\title{
Increased hippocampal accumulation of autophagosomes predicts short-term recognition memory impairment in aged mice
}

\author{
Virawudh Soontornniyomkij • Victoria B. Risbrough • Jared W. Young • \\ Benchawanna Soontornniyomkij • Dilip V. Jeste • Cristian L. Achim
}

Received: 23 January 2011 / Accepted: 2 March 2011 /Published online: 23 March 2011

(C) The Author(s) 2011. This article is published with open access at Springerlink.com

\begin{abstract}
Constitutive macroautophagy involved in the turnover of defective long-lived proteins and organelles is crucial for neuronal homeostasis. We hypothesized that macroautophagic dysregulation in selective brain regions was associated with memory impairment in aged mice. We used the single-trial object recognition test to measure short-term memory in 18 aged mice compared to 22 young mice and employed immunohistochemistry to assess cellular distribution of proteins involved in the selective degradation of ubiquitinated proteins via macroautophagy. Values of the discrimination ratio (DR, a measure of short-term recognition memory performance) in aged mice were significantly lower than those in young mice (median, 0.54 vs. $0.67 ; p=0.005, U$ test). Almost exclusively in aged mice, there were clusters of puncta immunoreactive for microtubule-associated protein 1 light chain 3 (LC3), ubiquitin- and LC3-binding protein
\end{abstract}

V. Soontornniyomkij $(\bowtie) \cdot$ V. B. Risbrough •

J. W. Young $\cdot$ B. Soontornniyomkij • D. V. Jeste

C. L. Achim

Sam and Rose Stein Institute for Research on Aging,

University of California, San Diego,

9500 Gilman Drive,

La Jolla, CA 92093-0603, USA

e-mail: vsoontor@ucsd.edu

V. Soontornniyomkij · V. B. Risbrough · J. W. Young •

B. Soontornniyomkij · D. V. Jeste $\cdot$ C. L. Achim

Department of Psychiatry, School of Medicine,

University of California, San Diego,

9500 Gilman Drive,

La Jolla, CA 92093-0603, USA p62, and ubiquitin in neuronal processes predominantly in the hippocampal formation, olfactory bulb/tubercle, and cerebellar cortex. The hippocampal burden of clustered puncta immunoreactive for LC3 and p62 exhibited inverse linear correlations with DR in aged mice ( $\rho=-0.48$ and $-0.55, p=0.044$ and 0.018 , respectively, Spearman's rank correlation). These findings suggest that increased accumulation of autophagosomes within neuronal processes in selective brain regions is characteristic of aging. The dysregulation of macroautophagy can adversely affect the turnover of aggregate-prone proteins and defective organelles, which may contribute to memory impairment in aged mice.

Keywords Autophagy · Brain aging · MAP1LC3 . Object recognition test $\cdot$ p $62 \cdot$ Ubiquitin

\section{Introduction}

The differential susceptibility of older individuals to the development of aging-related cognitive impairment may be driven by genetic polymorphisms, epigenetic phenomena, and dissimilar life-long environmental exposure to stressors (Goosens and Sapolsky 2007). While aging-related cognitive decline is different from well-characterized neurodegenerative disorders like Alzheimer's disease, the neurobiological substrates contributing to cognitive impairment in old age are still poorly understood. 
Among a variety of intracellular signaling pathways that have been implicated in aging, excessive stimulation of anabolic processes via the mammalian target of rapamycin (mTOR) kinase causes an imbalance that results in the accumulation of reactive oxygen species and the inhibition of macroautophagy, which in turn promote the development of agingrelated phenotypes (Cuervo 2008; García-Arencibia et al. 2010; Kamada et al. 2000; Levine and Kroemer 2008; Metcalf et al. 2010). In a mouse model of Alzheimer's disease, enhancement of macroautophagy via rapamycin-mediated inhibition of mTOR signaling ameliorated $\beta$-amyloid and Tau pathology and rescued cognitive impairment (Caccamo et al. 2010). The autophagic activity has been shown to decline with aging (Cuervo 2008; Levine and Kroemer 2008; Martinez-Vicente and Cuervo 2007). The autophagic-lysosomal system is a catalytic mechanism responsible for the turnover of dysfunctional organelles, membranes, and long-lived proteins (Cuervo et al. 2005; García-Arencibia et al. 2010). Macroautophagy, which is different from the other two pathways in this lysosome-dependent system (i.e., microautophagy and chaperone-mediated autophagy), is a regulated process in which portions of the cytoplasm are sequestered within double membrane-bound vesicles (autophagosomes) and subsequently transported to lysosomes (García-Arencibia et al. 2010; Levine and Kroemer 2008). The autophagosome is a globular organelle with a diameter of approximately $1 \mu \mathrm{m}$ (Ichimura and Komatsu 2010). Fusion of the autophagosome with the lysosome triggers the dissolution of the inner autophagosomal membrane and degradation of the cargo by lysosomal acid hydrolases (Mizushima et al. 2008).

In addition to stimulus-induced (adaptive) macroautophagy that responds to acute stress (e.g., nutrient starvation and growth factor withdrawal) by nonselectively degrading bulk cytoplasmic components, basal macroautophagy functions constitutively as a quality control system to ensure the turnover of damaged organelles and misfolded proteins (GarcíaArencibia et al. 2010; Ichimura and Komatsu 2010; Levine and Kroemer 2008). For instance, defective senescent mitochondria are sequestered within autophagosomes to prevent cell injury caused by the uncontrolled release of reactive oxygen species (Rami 2009). This homeostatic role of macroautophagy is particularly important for long-lived post-mitotic cells like neurons, which require efficient protein degradation to prevent accumulation of aggregate-prone proteins (Cuervo et al. 2005; Ravikumar et al. 2002). Furthermore, neurons are highly dependent on microtubulemediated axonal transport over long distances, including retrograde trafficking of autophagosomes toward the soma for lysosome-mediated degradation (Boland et al. 2008). Hence, it is likely that neurons are particularly vulnerable to macroautophagic dysregulation. Increased accumulation of autophagosomes in neurons has been described in diverse neurodegenerative disorders, such as Alzheimer's, Parkinson's, Huntington's, and motor neuron diseases (Cherra et al. 2010).

Microtubule-associated protein 1 light chain 3 (LC3) plays a crucial role in the formation of autophagosomes (Nakatogawa et al. 2007). Upon recruitment to the isolation membrane, cytosolic LC3 (LC3-I) is modified by conjugation with phosphatidylethanolamine into LC3-II, which is incorporated into the inner and outer autophagosomal membranes to regulate their elongation, curvature, and closure and function in cargo recruitment (García-Arencibia et al. 2010; Ichimura and Komatsu 2010). It was shown in neuronal cell cultures that upon induction of macroautophagy, the subcellular localization of LC3 redistributed from a diffuse cytoplasmic to a punctate pattern (Rami 2009). As LC3-II remains specifically associated with the completed autophagosome but not with other vesicular structures (García-Arencibia et al. 2010; Komatsu and Ichimura 2010; Levine and Kroemer 2008), the intracellular level of LC3-II has been widely used to estimate the burden of autophagosomes until they are degraded by lysosomal hydrolases (García-Arencibia et al. 2010; Rami 2009). In a mouse model of juvenile neuronal ceroid lipofuscinosis (Batten disease) caused by mutations in the endosomal/lysosomal membrane protein CLN3 gene, clusters of LC3-immunoreactive puncta were observed in the hippocampal CA2/CA3 and cerebellar cortex of 10-month-old homozygous $C \ln 3^{\Delta \text { ex7/8 }}$ knock-in mice, suggesting that autophagosome maturation that requires fusion with late lysosomes is disrupted in this mouse model (Cao et al. 2006). Ubiquitin- and LC3-binding protein p62 is particularly involved in the selective degradation of ubiquitinated proteins via macroautophagy (Kirkin et al. 2009; Metcalf et al. 2010). The p62 protein is localized to the autophagosome via interaction with LC3 and is degraded by the autophagic-lysosomal 
system. Notably, p62 has been identified as a component of cytoplasmic inclusions in Alzheimer's, Parkinson's, and motor neuron diseases (Ichimura and Komatsu 2010).

To study the relationship between memory impairment and macroautophagic dysregulation in aged mice, we assessed short-term non-spatial recognition memory by using the single-trial object recognition test (Young et al. 2009). We used immunohistochemistry to assess cellular distribution of autophagosomerelated proteins involved in selective degradation of ubiquitinated proteins via macroautophagy (i.e., LC3, p62, and ubiquitin) in the brain of aged mice compared to young mice. We hypothesized that macroautophagic dysregulation in selective brain regions was associated with memory impairment in aged mice. We found almost exclusively in the brain sections of aged mice clusters of puncta immunoreactive for the autophagosome-related proteins, predominantly in the hippocampal formation, olfactory bulb/tubercle, and cerebellar cortex.

\section{Materials and methods}

Animals and behavioral testing

Young (6 months old) and aged (26 months old) male $\mathrm{C} 57 \mathrm{BL} / 6 \mathrm{~N}$ mice were used for single-trial object recognition testing as previously described in detail (Soontornniyomkij et al. 2010). All procedures were in accordance with the "Principles of Laboratory Animal Care" (National Institutes of Health [NIH] publication no. 86-23, revised 1985) and approved by the University of California, San Diego Animal Care Committee. The ratio of the time spent exploring the novel object over the total amount of time spent exploring both novel and familiar objects during the retention phase of the object recognition test (i.e., [novel object exploration time]/[novel object+familiar object exploration time]), referred to as the discrimination ratio (DR) (Bevins and Besheer 2006), was used to measure the short-term non-spatial recognition memory performance.

\section{Brain histology}

There were 18 aged mice and 22 young mice examined, the right hemi-brains of which were included in the immunohistologic study. The frontal lobe and olfactory bulb of one of the young mice were not available for the present study. Fivemicrometer-thick formalin-fixed paraffin-embedded parasagittal sections of all the hemi-brains revealed neuroanatomic landmarks consistent with the levels from 1,250 to $1,750 \mu \mathrm{m}$ from the midline (Valverde 1998). No significant histopathologic changes were observed in any of the hemi-brain sections stained with hematoxylin and eosin.

\section{Immunohistochemistry}

To evaluate the cellular distribution of autophagosomerelated proteins, we used primary antibodies raised against LC3 (rabbit polyclonal, APG8b, \#AP1802a, Abgent, San Diego, CA, USA, 1:50 dilution), p62 (rabbit polyclonal, SQSTM1, \#AP2183b, Abgent, 1:25), and ubiquitin (mouse monoclonal, \#MAB1510, Millipore, Temecula, CA, USA, 1:4,000). The anti-LC3 antibody has been shown to recognize both LC3-I and LC3-II on Western blots (Cao et al. 2006). Microtubule-associated proteins (MAP) were identified using anti-MAP2 (goat polyclonal, \#sc-12012, Santa Cruz Biotechnology, Santa Cruz, CA, USA, 1:100) and anti-phosphorylated Tau (phospho-Thr-231, rabbit polyclonal, \#A00406, GenScript, Piscataway, NJ, USA, 1:200) antibodies. For presynaptic proteins, we used anti-synapsin IIa (rabbit polyclonal, \#sc-25538, Santa Cruz Biotechnology, 1:100) and anti-synaptophysin (rabbit polyclonal, \#A0010, DakoCytomation, Carpinteria, CA, USA, 1:200) antibodies. Antigen retrieval was carried out by autoclaving at $121^{\circ} \mathrm{C}$ for $20 \mathrm{~min}$ with $10 \mathrm{mM}$ Tris/ $1 \mathrm{mM}$ EDTA-2Na/0.05\% Tween 20 buffer (pH 9) for LC3, p62, MAP2, synapsin IIa, and synaptophysin, and with $10 \mathrm{mM}$ sodium citrate $/ 0.05 \%$ Tween 20 buffer (pH 6) for ubiquitin and p-Tau. Immunohistochemical signals were developed using the species-appropriate ImmPRESS ${ }^{\mathrm{TM}}$ anti-Ig (peroxidase) polymer detection kits (Vector Laboratories, Burlingame, CA, USA) and diaminobenzidine (ImmPACT TM DAB peroxidase substrate, Vector Laboratories), as previously described (Soontornniyomkij et al. 2010). For the negative reagent control, the primary antibody was omitted. For each of the proteins studied, all the hemi-brain sections were processed in the same batch.

To document whether clustered autophagic puncta were localized to neuronal processes, we performed double immunofluorescent staining with four anti- 
body pairings: MAP2 (mouse monoclonal, \#M4403, Sigma-Aldrich, Saint Louis, MO, USA, 1:200)/LC3 (1:5), MAP2 (\#M4403, Sigma-Aldrich, 1:200)/p62 (1:10), ubiquitin $(1: 2,000) /$ MAP2 (rabbit polyclonal, \#PRB-547C, Covance, Princeton, NJ, USA, 1:200), and ubiquitin (1:2,000)/synapsin IIa (1:50). Double immunofluorescent staining was also conducted to confirm the localization of ubiquitin within autophagosomes with ubiquitin $(1: 2,000) / \mathrm{LC} 3 \quad(1: 5)$ and ubiquitin $(1: 2,000) /$ p62 (1:10) antibody pairings. Antigen retrieval was performed by autoclaving at $121^{\circ} \mathrm{C}$ for 20 min either with $10 \mathrm{mM}$ sodium citrate/ $0.05 \%$ Tween 20 buffer (pH 6) or $10 \mathrm{mM}$ Tris $/ 1 \mathrm{mM}$ EDTA-2Na/0.05\% Tween 20 buffer (pH 9). Because primary antibodies in each pairing used were raised in different animal species, tissue sections were incubated with a mixture of the primary antibody pairing at $4^{\circ} \mathrm{C}$ for $24 \mathrm{~h}$, and then with the corresponding mixture of Alexa Fluor 488 and Alexa Fluor 568 conjugated anti-IgG secondary antibodies (Invitrogen, Carlsbad, CA, USA, 1:200) at room temperature for $2 \mathrm{~h}$. For the negative control, the primary antibody pairing was omitted. The tissue sections were mounted with VECTASHIELD ${ }^{\circledR}$ Hard Set ${ }^{\mathrm{TM}}$ with DAPI mounting medium (Vector Laboratories) and qualitatively examined by DeltaVision ${ }^{\circledR}$ RT deconvolution microscopy (Applied Precision, Issaquah, WA, USA).

\section{Semiquantification of clusters of immunoreactive puncta}

The entire hemi-brain sections immunostained with DAB were digitally scanned using a microscope slide scanner (Aperio ScanScope ${ }^{\circledR}$ GL, Vista, CA, USA) equipped with a $20 \times$ objective lens (yielding the resolution of $0.5 \mu \mathrm{m}$ per pixel). The digital slides were viewed on a high-definition 24-inch LCD TFT monitor using the ImageScope ${ }^{\mathrm{TM}}$ software (Aperio) at $10 \times$ and $20 \times$ zooms. In each of the entire dorsal hippocampal formation (i.e., the hippocampus proper, dentate gyrus, and subiculum), olfactory bulb/tubercle, and cerebellar cortex, the number of clusters of immunoreactive puncta was independently recorded by manually counting by two of the authors (V. S. and C. L. A.) who were blinded to the DR values. Single immunoreactive puncta without cluster formation were not recorded in the present study. The semiquantitative burden of clustered immunoreactive puncta was classified into none ( 0 clusters), sparse $(1-3)$, moderate (4-
10), and abundant (11 or greater) in each of the three brain regions.

For illustration, composite digital images (Fig. 1a-1) were obtained from the Aperio digital slides. The inset images (in Fig. 1a-g, j) were taken with a $40 \times$ objective lens on an Olympus BX40 microscope equipped with a Nikon Digital Sight DS-5M digital camera.

Statistical analysis

The Mann-Whitney $U$ test was used to compare continuous variables between two independent groups. The Spearman's rank correlation $(\rho)$ test was employed to evaluate the linear relationship between two continuous variables in a given group. To determine associations among categorical proportions, the Chi-square $\left(\chi^{2}\right)$ test was used. The GraphPad InStat 3 for Macintosh software (GraphPad Software, La Jolla, CA, USA) was used to perform all statistical analysis. All $p$ values calculated were two-tailed and considered statistically significant at a threshold of $p<0.05$.

\section{Results}

Clustered immunoreactive puncta in selective brain regions

On hemi-brain sections immunostained for LC3, p62, ubiquitin, MAP2, and synapsin IIa, there were clusters of immunoreactive puncta unevenly distributed in the dorsal hippocampal formation (predominantly in the neuropil subfields, Fig. 1a-c, e, f), olfactory bulb/ tubercle (Fig. 1d), and cerebellar cortex (Purkinje cell and granule cell layers, Fig. 1g) particularly of aged mice (Table 1). Whether there were clustered puncta immunoreactive for ubiquitin and synapsin IIa in the cerebellar cortex was undetermined because of intense immunoreactivity for these two proteins in the granule cell layer. Only one aged mouse that showed the highest numbers of clusters of puncta immunoreactive for LC3, p62, ubiquitin, MAP2, and synapsin IIa in the hippocampal formation also had a few clusters of puncta immunoreactive for LC3 and p62 in the thalamus. There were no clustered immunoreactive puncta observed in other brain regions, such as the cerebral cortex, caudate-putamen, globus pallidus, hypothalamus, midbrain, and brainstem. No clustered puncta were seen in any brain regions on the sections 

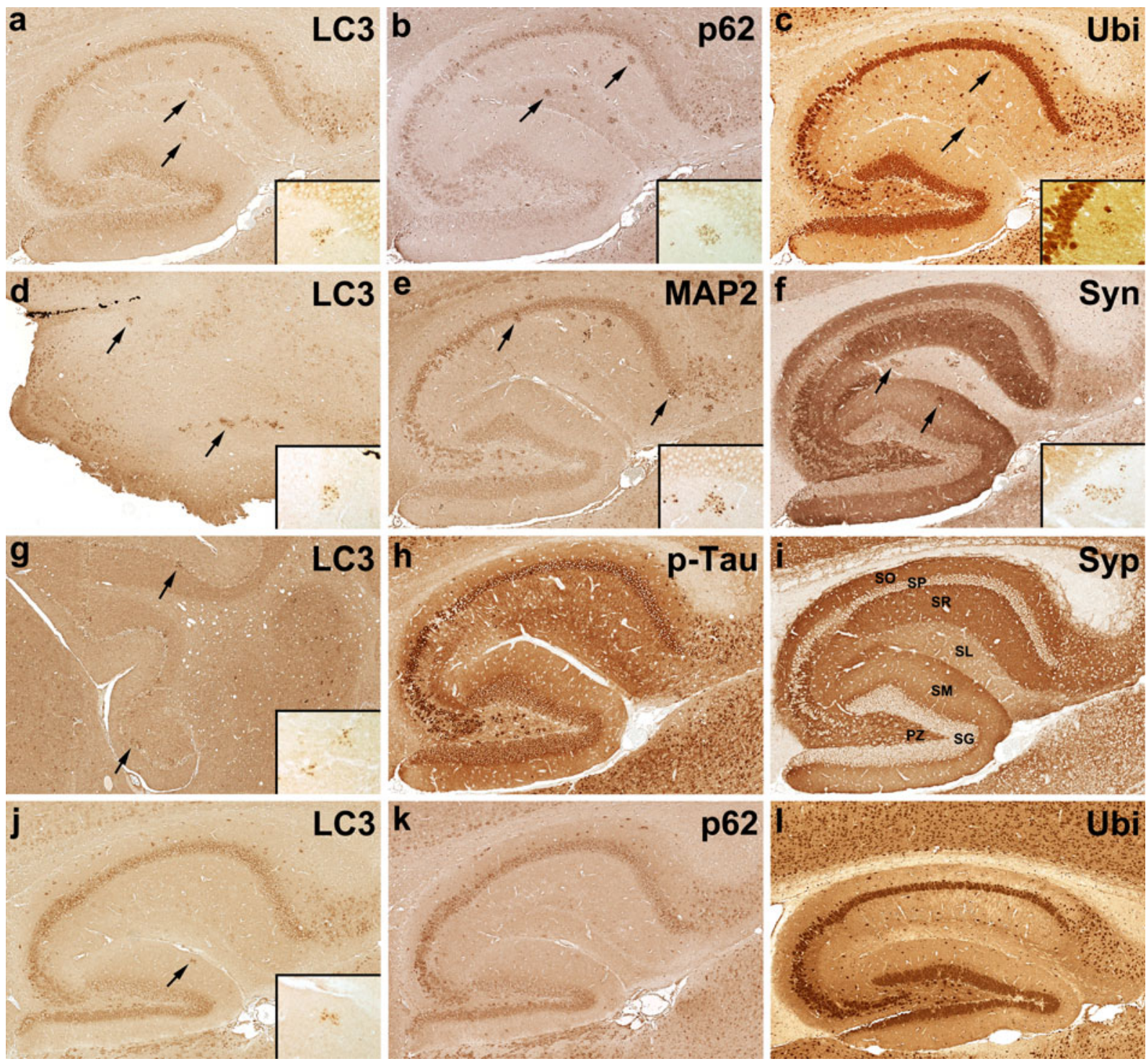

Fig. 1 Brain sections of aged mice $(\mathbf{a}-\mathbf{i})$ are depicted in comparison with those of young mice $(\mathbf{j}-\mathbf{l})$. In the hippocampal formation of aged mice, there are scattered clusters of puncta (arrows, higher magnification inset) immunoreactive for microtubule-associated protein 1 light chain $3(L C 3, \mathbf{a})$, p62 (b), ubiquitin $(U b i, \mathbf{c})$, microtubule-associated protein $2(M A P 2, \mathbf{e})$, and synapsin IIa (Syn, f). These immunoreactive puncta (arrows, higher magnification inset) are also present in the olfactory bulb (d) and cerebellar cortex (g). No clusters of immunoreactive puncta are observed on any of the hemi-brain sections immunos-

immunostained for p-Tau or synaptophysin (Fig. 1h, i; Table 1). In contrast to aged mice, their young counterparts showed no or sparse clusters of immunoreactive puncta on hemi-brain sections studied (Fig. $1 \mathrm{j}-1)$. tained for phosphorylated Tau $(p-T a u, \mathbf{h})$ or synaptophysin $(S y p, \mathbf{i})$. Note that hippocampal formation sections $(\mathbf{a}-\mathbf{c}, \mathbf{e}, \mathbf{f}, \mathbf{h}, \mathbf{i})$ are of one aged mouse, and olfactory bulb (d) and cerebellar (g) sections are of another aged mouse. By contrast, young mice $(\mathbf{j}-\mathbf{l}$, the hippocampal formation of one young mouse) show no $(\mathbf{k}, \mathbf{l})$ or sparse (j [arrow], higher magnification inset) clusters of immunoreactive puncta. $S O$ stratum oriens, $S P$ stratum pyramidale, $S R$ stratum radiatum, $S L$ stratum lacunosum-moleculare, $S M$ stratum moleculare, $S G$ stratum granulosum, $P Z$ pleomorphic zone (hilus)

Clustered immunoreactive puncta localized to neuronal processes

On double immunofluorescent staining, MAP2 was co-localized within clustered puncta with LC3, p62, 
Table 1 Distributions of clustered immunoreactive puncta in the hippocampal subfields, olfactory bulb/tubercle, and cerebellar cortex

\begin{tabular}{|c|c|c|c|c|c|c|c|c|c|c|c|c|c|c|}
\hline & \multicolumn{4}{|c|}{ Hippocampal CA1 } & \multicolumn{4}{|c|}{ Hippocampal CA2/CA3 } & \multicolumn{3}{|c|}{ Dentate gyrus } & \multirow[t]{2}{*}{ Subiculum } & \multirow[t]{2}{*}{ Olfactory bulb/tubercle } & \multirow[t]{2}{*}{ Cerebellar cortex } \\
\hline & SL & SR & SP & $\mathrm{SO}$ & SL & SR & $\mathrm{SP}$ & $\mathrm{SO}$ & $\mathrm{SM}$ & SG & $\mathrm{PZ}$ & & & \\
\hline LC3 & + & + & + & + & + & + & - & + & + & - & - & + & + & + \\
\hline p62 & + & + & + & + & - & + & + & + & + & + & - & + & + & + \\
\hline Ubiquitin & + & + & - & + & + & + & - & + & + & - & + & + & + & Undetermined $^{\mathrm{a}}$ \\
\hline MAP2 & + & + & + & + & - & + & + & + & + & + & - & + & + & + \\
\hline p-Tau & - & - & - & - & - & - & - & - & - & - & - & - & - & - \\
\hline Synapsin IIa & + & + & + & + & - & - & + & - & + & - & - & + & + & Undetermined $^{\mathrm{a}}$ \\
\hline Synaptophysin & - & - & - & - & - & - & - & - & - & - & - & - & - & - \\
\hline
\end{tabular}

$L C 3$ microtubule-associated protein 1 light chain 3, MAP2 microtubule-associated protein 2, $p$-Tau phosphorylated Tau, $S L$ stratum lacunosum-moleculare, $S R$ stratum radiatum, $S P$ stratum pyramidale, $S O$ stratum oriens, $S M$ stratum moleculare, $S G$ stratum granulosum, $P Z$ pleomorphic zone (hilus), + present, - absent

${ }^{a}$ Whether there were clustered puncta immunoreactive for ubiquitin and synapsin IIa in the cerebellar cortex was undetermined due to intense immunoreactivity of these two proteins in the granule cell layer

and ubiquitin (Fig. 2a-i). Ubiquitin was also colocalized within clustered puncta with synapsin IIa (Fig. 2j-1). These findings suggest that clustered puncta are localized to neuronal processes. In addition, ubiquitin was co-localized within clustered puncta with LC3 and p62 (Fig. 2m-r), confirming the localization of ubiquitin within autophagosomes.

Burden of clustered immunoreactive puncta in aged vs. young mice

Semiquantitative analysis of the burden of clustered immunoreactive puncta in the hippocampal formation of all 40 mice (i.e., 22 young and 18 aged mice) revealed a highly significant linear trend toward greater load in aged mice ( $p<0.0001$ for each of LC3, p62, ubiquitin, MAP2, and synapsin IIa stainings; $\chi^{2}$ test for linear trend [Fig. 3a-e]). Only a small minority of young mice showed clustered immunoreactive puncta, the quantities of which were sparse at most. In other words, these clustered immunoreactive puncta were observed almost exclusively in the aged mouse brain. Similarly, the burden of clustered immunoreactive puncta in the olfactory bulb/tubercle (21 young and 18 aged mice) showed a highly significant trend toward greater load in aged mice $(p<0.0001$ for each of LC3, p62, and ubiquitin stainings; $p=0.0002$ for each of MAP2 and synapsin IIa stainings; $\chi^{2}$ test for linear trend), as did the burden in the cerebellar cortex (22 young and 18 aged mice, $p<0.0001$ for each of LC3, p62, and MAP2 stainings; $\chi^{2}$ test for linear trend).
Correlation between numbers of clusters of immunoreactive puncta in aged mice

The number of clusters of puncta immunoreactive for autophagosome-related proteins (i.e., LC3, p62, and ubiquitin) correlated significantly with each other in the hippocampal formation, olfactory bulb/tubercle, and cerebellar cortex of aged mice (Fig. 4). Similarly, the number of clusters of MAP2 puncta correlated significantly with those of LC3, p62, and ubiquitin in all the three brain regions (Fig. 4). In addition, there was a significant correlation between the number of clusters of synapsin IIa puncta and that of LC3 in the hippocampal formation (Fig. 4).

Correlation between number of clusters

of immunoreactive puncta and memory performance

On analysis of object recognition testing, the DR distribution in aged mice was significantly lower than that in young mice (median, 0.54 vs. 0.67 ; interquartile range, 0.11 vs. $0.19 ; p=0.005, U$ test).

As evidence has suggested that the hippocampus, but not the olfactory bulb or cerebellum, is essential for object recognition memory performance in rodents (Brown et al. 2010; Young et al. 2009), we specifically examined the correlation between the hippocampal burden of clustered immunoreactive puncta and DR. In the hippocampal formation of aged mice, the numbers of clusters of puncta immunoreactive for LC3 and p62 exhibited significant inverse correlations 
Fig. 2 Double immunofluorescent staining of the hippocampal formation of aged mice shows within clustered puncta co-localization of microtubule-associated protein $2(M A P 2, \mathbf{a}, \mathbf{d}, \mathbf{h})$ with microtubule-associated protein 1 light chain $3(L C 3, \mathbf{b}$, CA1 stratum radiatum), p62 (e, stratum moleculare), and ubiquitin (Ubi, g, CA1 stratum lacunosum-moleculare [upper] and stratum moleculare [lower ]). Ubiquitin $(\mathbf{j}, \mathbf{m}, \mathbf{p})$ is also co-localized within clustered puncta with synapsin IIa (Syn, k [arrows], CA1 stratum lacunosummoleculare), LC3 (n, CA1 stratum radiatum), and p62 (q, stratum moleculare). c, f, $\mathbf{i}, \mathbf{l}, \mathbf{0}, \mathbf{r}$ Each represents a merged image in their respective row, with DAPIlabeled nuclei (blue) in c, $\mathbf{f}$, and $\mathbf{i}$; $b a r=20 \mu \mathrm{m}$. Note that $\mathbf{a}-\mathbf{i}$ were obtained from one aged mouse and $\mathbf{j}-\mathbf{r}$ from another aged mouse a

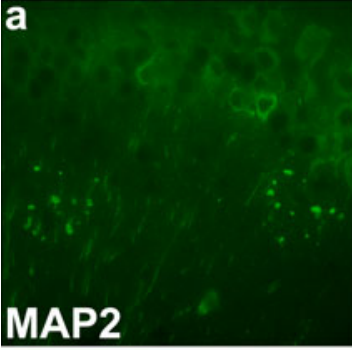

c

\section{MAP2}

g

Ubi

j

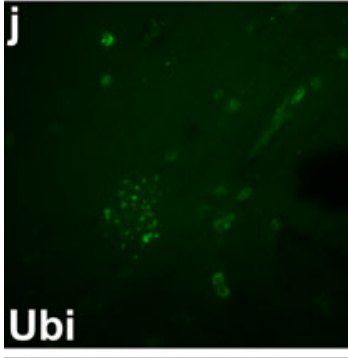

m

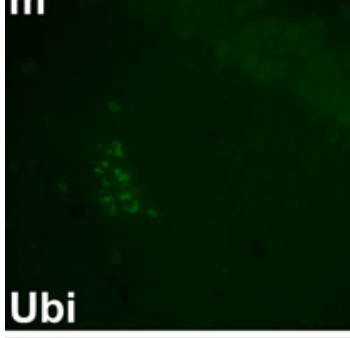

p

Ubi
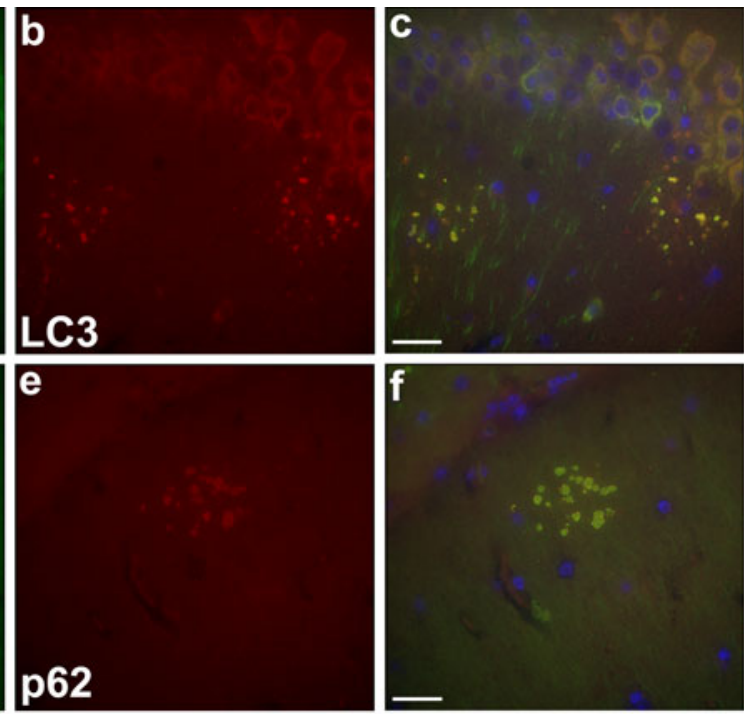

h

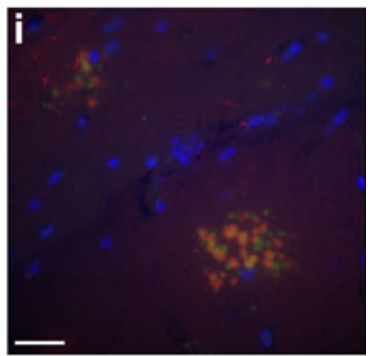

MAP2
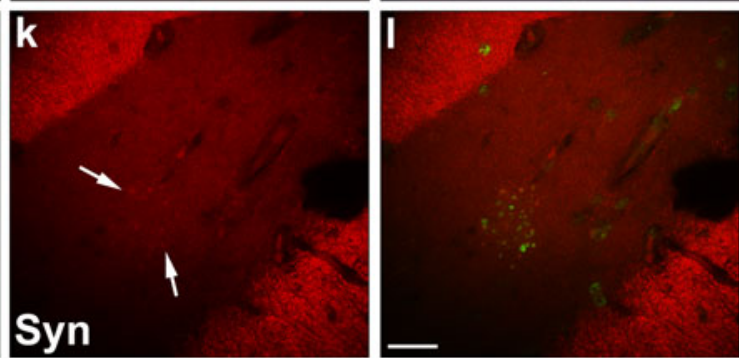

n

0

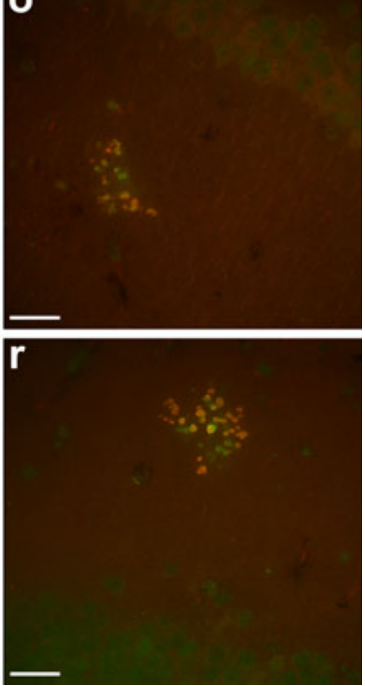



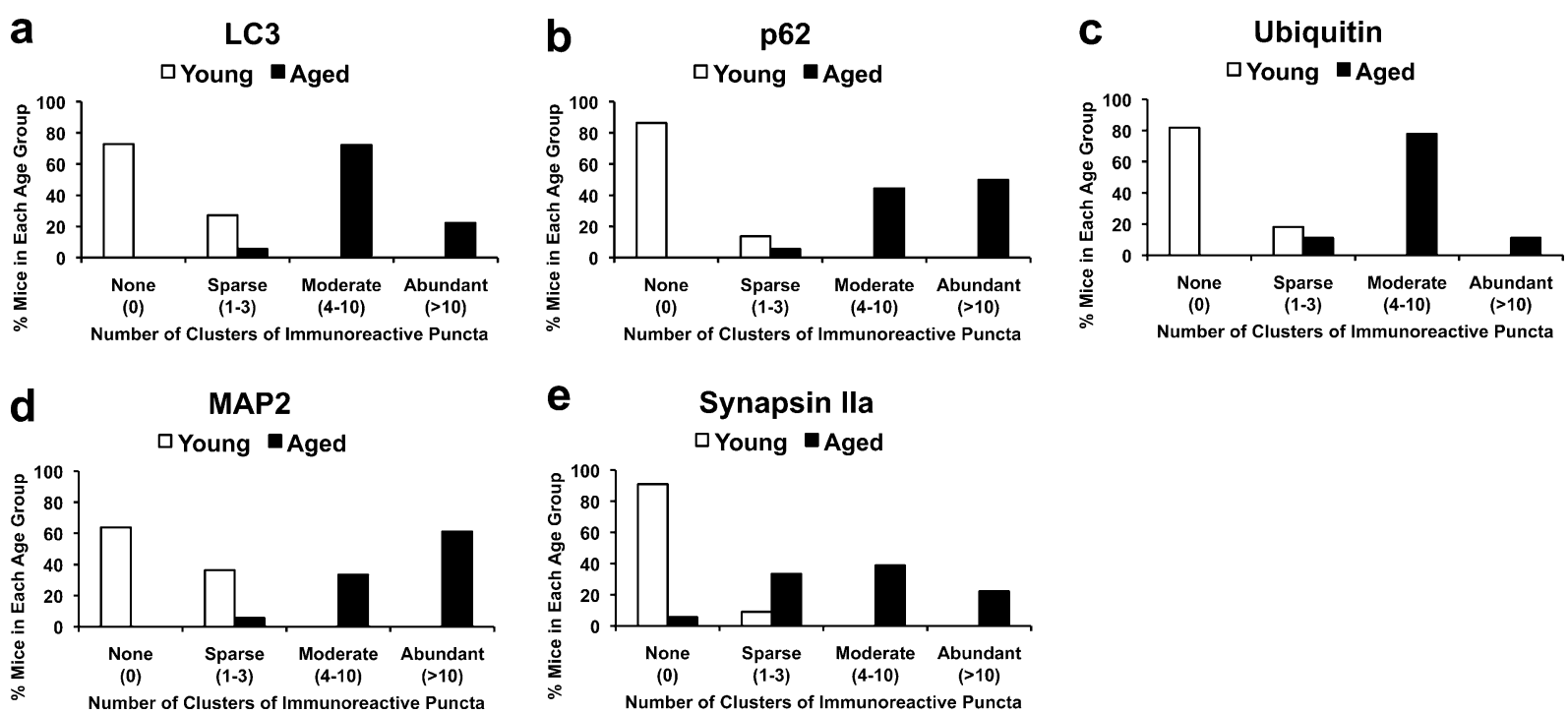

Fig. 3 In the hippocampal formation of all mice studied (22 young and 18 aged), the semiquantitative burden of clustered puncta immunoreactive for microtubule-associated protein 1 light chain 3 ( $L C 3$, a), p62 (b), ubiquitin (c), microtubule- associated protein $2(M A P 2$, d), and synapsin IIa (e) each shows a significant linear trend toward greater load in aged mice $(p<0.0001$, Chi-square test for linear trend)

\section{Discussion}

In the present study, clustered puncta immunoreactive for autophagosome-related proteins in selective regions of the mouse brain were similar to the autophagic puncta reported by Cao et al. (2006) in the hippocampal CA2/CA3 and cerebellar cortex of reactive for MAP2 or synapsin IIa.

\section{Correlation between Numbers of Clusters of Immunoreactive Puncta in Aged Mice}

- Hippocampus $\square$ Olfactory $\square$ Cerebellum

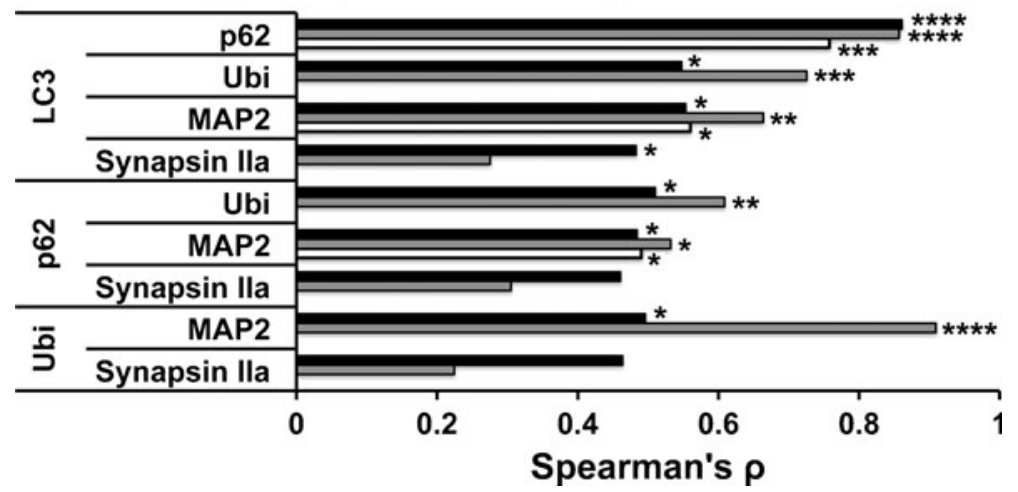

Fig. 4 The linear correlation coefficient $(\rho)$ between the numbers of clusters of immunoreactive puncta is shown for each pair of proteins studied in each of three brain regions (i.e., the hippocampal formation, olfactory bulb/tubercle, and cerebellar cortex) of 18 aged mice $(* p<0.05, * * p<0.01, * * * p<$ $0.001, * * * * p<0.0001$, Spearman's rank correlation). Note whether there were clustered puncta immunoreactive for ubiquitin and synapsin IIa in the cerebellar cortex was undetermined due to intense immunoreactivity of these two proteins in the granule cell layer. $L C 3$ microtubule-associated protein 1 light chain 3, Ubi ubiquitin, MAP2 microtubuleassociated protein 2 


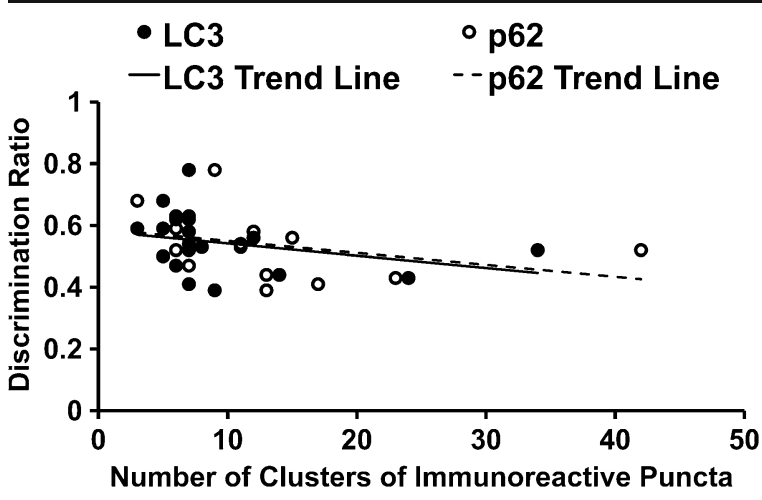

Fig. 5 Scattergraphs with their trend lines show significant inverse correlations between the discrimination ratio (i.e., the ratio of the time spent exploring the novel object over the total amount of time spent exploring both novel and familiar objects during the retention phase of the single-trial object recognition test) as a measure of the short-term recognition memory performance and the number of clusters of puncta immunoreactive for microtubule-associated protein 1 light chain $3(L C 3)$ and p62 in the hippocampal forma tion of 18 aged mice $(\rho=-0.48$ and $-0.55, p=0.044$ and 0.018 , respectively, Spearman's rank correlation)

homozygous $C \ln 3^{\Delta \mathrm{ex} 7 / 8}$ knock-in mice as a model of juvenile neuronal ceroid lipofuscinosis, using the same anti-LC3 antibody. We found increased accumulation of autophagosomes predominantly in three distinct brain regions (i.e., the hippocampal formation, olfactory bulb/tubercle, and cerebellar cortex), suggesting that these neuroanatomic regions are more vulnerable to macroautophagic dysregulation compared to others. The considerably greater burden of observed autophagosomes in the brains of aged mice compared to young mice suggests that increased accumulation of autophagosomes (involved in the p62-mediated selective degradation of ubiquitinated proteins via macroautophagy) in selective brain regions is characteristic of aging.

Recent studies using mice deficient for autophagyrelated proteins (e.g., Atg5 and Atg7) specifically in the central nervous system (CNS) have demonstrated the importance of constitutive macroautophagy in neuronal homeostasis (Hara et al. 2006; Komatsu et al. 2006; Metcalf et al. 2010). Dysregulation of macroautophagy may promote the intracellular accumulation of defective organelles and aggregate-prone proteins, which can disturb neuronal function and lead to neurodegeneration (Cherra et al. 2010; Levine and Kroemer 2008; Metcalf et al. 2010; Moreira et al. 2010). In support of this notion, the present study demonstrated significant linear correlation between the burden of autophago- some accumulation (represented by the numbers of clusters of puncta immunoreactive for LC3 and p62) in the hippocampal formation and the short-term recognition memory impairment in aged mice. This finding can be considered biologically relevant, as a large body of evidence has suggested that the object recognition memory involves the hippocampus and perirhinal cortex, as well as the prefrontal cortex (Brown et al. 2010; Young et al. 2009). We did not observe clustered immunoreactive puncta in the cerebral cortex. The range of parasagittal hemi-brain sections examined in our study did not include the perirhinal cortex.

Increased autophagosome accumulation in neurons may be due either to enhanced assembly or diminished degradation of autophagosomes or both (Chu 2006; Levine and Kroemer 2008). Increased autophagosome formation usually occurs in the adaptive response to stress signals, such as nutrient starvation, deficient insulin signaling, and mTOR inactivation; however, CNS neurons are relatively protected from experiencing nutrient starvation in vivo. Decreased autophagosome degradation is associated with unsuccessful completion of macroautophagy (e.g., impaired fusion with lysosomes and decreased lysosomal proteolysis), which may result in adverse pathophysiological consequences, such as those seen in aging and neurodegeneration (Levine and Kroemer 2008). Within neurons, autophagosomes are formed randomly in synaptic terminals and along neuronal processes, are then centripetally transported along microtubules in a dynein-dependent manner toward the soma, where lysosomes are clustered around the microtubule-organizing center located near the nucleus, and finally mature to autophagolysosomes by fusing with lysosomes (Chu 2006; García-Arencibia et al. 2010; Nixon 2007). In an in vivo study using transgenic mice expressing a fluorescent autophagosome marker (GFP-LC3), LC3 in healthy neurons existed predominantly as soluble LC3-I and the level of membrane-bound LC-II did not increase after nutrient starvation, in contrast to the liver and heart (Mizushima et al. 2004). Neurons, different from most other cell types, can uptake glucose independently of insulin. In the liver, starvation-induced macroautophagy functions in part to supply the CNS with fuel via amino acid deamination and conversion to glucose. Accordingly, adaptive macroautophagic responses in neurons may be involved more in neuritic remodeling than in supplying amino acids and other substrates for energy production (Chu 2006). 
Ultrastructural analysis confirmed the paucity of autophagosomes in healthy neurons (Nixon et al. 2005). This may be attributed to the high efficiency of constitutive macroautophagy in healthy neurons with highly dynamic vesicular trafficking in keeping balance between formation and clearance of autophagosomes (Boland et al. 2008; Chu 2006). Still, an alternative interpretation states that neurons normally maintain low levels of autophagosome biosynthesis (Moreira et al. 2010). In the Alzheimer's disease brain, there is massive accumulation of autophagosomes within neuritic processes and synaptic terminals (Nixon et al. 2005). In addition to increased induction of macroautophagy, in vitro studies using primary cortical neurons have suggested deficiencies in the retrograde transport and maturation of autophagosomes, resulting in prolongation of their half-life, as another potential mechanism of increased autophagosome accumulation observed in neuronal processes in Alzheimer's disease (Boland et al. 2008; Nixon 2007).

As aging-related decline in macroautophagic efficiency in almost all organisms and tissues analyzed has been described to be primarily associated with deficiencies in autophagosome formation, fusion with lysosomes, and lysosome-dependent degradation (Cuervo 2008; Levine and Kroemer 2008; MartinezVicente and Cuervo 2007), it is possible that in the present study the aging-related finding of increased autophagosome accumulation in selective regions of the mouse brain is attributed to macroautophagic dysregulation including retarded retrograde axonal transport and defective degradation of autophagosomes (Nixon 2007). In a mouse model of Alzheimer's disease, early axonopathy and axonal transport deficits were shown to precede the well-established diseaserelated pathology (Stokin et al. 2005). Accordingly, therapeutic interventions by stimulating autophagosome formation (e.g., treatments with rapamycin to inhibit mTOR signaling and with inositol-reducing agents like lithium involved in mTOR-independent activation of macroautophagy) may not be worthwhile unless impediments to effective axonal trafficking, maturation, and lysosomal degradation of autophagosomes are also removed (GarcíaArencibia et al. 2010; Metcalf et al. 2010; Sarkar and Rubinsztein 2008).

In our study, the strong direct correlation between the number of clusters of LC3 puncta and that of p62 (Fig. 4) suggests that these structures are involved in the p62-mediated selective degradation of ubiquitinated proteins via macroautophagy (Kirkin et al. 2009). In addition, the fact that MAP2 was colocalized with LC3, p62, and ubiquitin within clustered puncta suggests that the observed LC3immunoreactive autophagosomes are localized to neuronal processes and that MAP2 undergoes turnover by means of the selective degradation of ubiquitinated proteins via macroautophagy. We did not observe any clusters of puncta immunoreactive for p-Tau (Thr-231) or synaptophysin in any hemibrain sections examined, suggesting that these two proteins may not use macroautophagy as their primary recycling process in vivo. Tau contains a pentapeptide motif biochemically related to KFERQ in its amino acid sequence and, when it is in a soluble form and amendable to complete unfolding, can be a potential substrate for chaperone-mediated autophagy. However, the selective clearance of cytosolic proteins via chaperone-mediated autophagy is low under basal conditions in most cells but is maximally activated in response to stress (Koga and Cuervo 2010). Although recent studies using cell models showed that fulllength wild-type Tau was preferentially degraded by macroautophagy (Hamano et al. 2008; Wang et al. 2009), the role of constitutive macroautophagy in recycling of soluble Tau in vivo remains controversial, and the state of phosphorylation may also determine the effective pathway in which Tau is degraded (Wang et al. 2010). Evidence has suggested that the crosstalk between macroautophagy and chaperone-mediated autophagy operates bi-directionally to ensure that deficiency in one pathway triggers compensatory activation of the other (Kaushik et al. 2008; Koga and Cuervo 2010).

In summary, our findings suggest that increased accumulation of autophagosomes within neuronal processes in specific brain regions is characteristic of aging. This dysregulation of macroautophagy may adversely affect the turnover of aggregate-prone proteins, which can disturb neuronal function (Cherra et al. 2010; Levine and Kroemer 2008; Moreira et al. 2010). Our results also show that the burden of autophagosome accumulation in the hippocampal formation predicts short-term recognition memory impairment in aged mice. Yet, this conclusion may not be generalized to other domains of cognitive impairment that were not evaluated in the current study. We studied parasagittal hemi-brain sections at 
the levels from 1,250 to $1,750 \mu \mathrm{m}$ from the midline and observed clustered immunoreactive puncta predominantly in the hippocampal formation, olfactory bulb/tubercle, and cerebellar cortex and rarely in the thalamus. Therefore, we cannot exclude the possibility of increased accumulation of autophagosomes in other brain regions not included in this series of analysis. Similar studies in the perirhinal cortex in particular are warranted as this neuroanatomic region, in addition to the hippocampus, has been shown to play a role in object recognition memory performance (Brown et al. 2010; Young et al. 2009).

Acknowledgments This work was supported in part by a Sam and Rose Stein Institute for Research on Aging fellowship (J. W. Young), a junior faculty pilot award (V. B. Risbrough), the Veterans Affairs Center of Excellence for Stress and Mental Health (V. B. Risbrough), the NIH grant MH052885 (V. B. Risbrough), and the NIH grants DA26306, MH83506, MH62512, MH81482, and HNRC889 (C. L. Achim and V. Soontornniyomkij). DeltaVision ${ }^{\circledR}$ RT deconvolution microscopy was supported by the University of California, San Diego Neuroscience Microscopy Shared Facility grant P30 NS047101.

Disclosure There are no real or potential conflicts of interest for any of the authors to disclose.

Open Access This article is distributed under the terms of the Creative Commons Attribution Noncommercial License which permits any noncommercial use, distribution, and reproduction in any medium, provided the original author(s) and source are credited.

\section{References}

Bevins RA, Besheer J (2006) Object recognition in rats and mice: a one-trial non-matching-to-sample learning task to study 'recognition memory'. Nat Protoc 1:1306-1311

Boland B, Kumar A, Lee S, Platt FM, Wegiel J, Yu WH, Nixon RA (2008) Autophagy induction and autophagosome clearance in neurons: relationship to autophagic pathology in Alzheimer's disease. J Neurosci 28:6926-6937

Brown MW, Warburton EC, Aggleton JP (2010) Recognition memory: material, processes, and substrates. Hippocampus 20:1228-1244

Caccamo A, Majumder S, Richardson A, Strong R, Oddo S (2010) Molecular interplay between mammalian target of rapamycin (mTOR), amyloid-beta, and Tau: effects on cognitive impairments. J Biol Chem 285:1310713120

Cao Y, Espinola J, Fossale E, Massey A, Cuervo A, MacDonald M, Cotman S (2006) Autophagy is disrupted in a knock-in mouse model of juvenile neuronal ceroid lipofuscinosis. J Biol Chem 281:20483-20493

Cherra SJ, Dagda RK, Chu CT (2010) Review: autophagy and neurodegeneration: survival at a cost? Neuropathol Appl Neurobiol 36:125-132

Chu CT (2006) Autophagic stress in neuronal injury and disease. J Neuropathol Exp Neurol 65:423-432

Cuervo AM (2008) Autophagy and aging: keeping that old broom working. Trends Genet 24:604-612

Cuervo A, Bergamini E, Brunk U, Dröge W, Ffrench M, Terman A (2005) Autophagy and aging: the importance of maintaining "clean" cells. Autophagy 1:131-140

García-Arencibia M, Hochfeld WE, Toh PP, Rubinsztein DC (2010) Autophagy, a guardian against neurodegeneration. Semin Cell Dev Biol 21:691-698

Goosens KA, Sapolsky RM (2007) Stress and glucocorticoid contributions to normal and pathological aging. In: Riddle DR (ed) Brain aging: models, methods, and mechanisms. CRC, New York, pp 305-314

Hamano T, Gendron TF, Causevic E et al (2008) Autophagiclysosomal perturbation enhances tau aggregation in transfectants with induced wild-type tau expression. Eur J Neurosci 27:1119-1130

Hara T, Nakamura K, Matsui M et al (2006) Suppression of basal autophagy in neural cells causes neurodegenerative disease in mice. Nature 441(7095):885-889

Ichimura Y, Komatsu M (2010) Selective degradation of $\mathrm{p} 62$ by autophagy. Semin Immunopathol 32:431-436

Kamada Y, Funakoshi T, Shintani T, Nagano K, Ohsumi M, Ohsumi Y (2000) Tor-mediated induction of autophagy via an Apg1 protein kinase complex. J Cell Biol 150:1507-1513

Kaushik S, Massey AC, Mizushima N, Cuervo AM (2008) Constitutive activation of chaperone-mediated autophagy in cells with impaired macroautophagy. Mol Biol Cell 19:21792192

Kirkin V, McEwan DG, Novak I, Dikic I (2009) A role for ubiquitin in selective autophagy. Mol Cell 34:259-269

Koga H, Cuervo AM (2010) Chaperone-mediated autophagy dysfunction in the pathogenesis of neurodegeneration. Neurobiol Dis. doi:10.1016/j.nbd.2010.07.006

Komatsu M, Ichimura Y (2010) Selective autophagy regulates various cellular functions. Genes Cells 15:923-933

Komatsu M, Waguri S, Chiba T et al (2006) Loss of autophagy in the central nervous system causes neurodegeneration in mice. Nature 441(7095):880-884

Levine B, Kroemer G (2008) Autophagy in the pathogenesis of disease. Cell 132:27-42

Martinez-Vicente M, Cuervo AM (2007) Autophagy and neurodegeneration: when the cleaning crew goes on strike. Lancet Neurol 6:352-361

Metcalf DJ, García-Arencibia M, Hochfeld WE, Rubinsztein DC (2010) Autophagy and misfolded proteins in neurodegeneration. Exp Neurol. doi:10.1016/j.expneurol.2010.11.003

Mizushima N, Yamamoto A, Matsui M, Yoshimori T, Ohsumi Y (2004) In vivo analysis of autophagy in response to nutrient starvation using transgenic mice expressing a fluorescent autophagosome marker. Mol Biol Cell 15:1101-1111

Mizushima N, Levine B, Cuervo AM, Klionsky DJ (2008) Autophagy fights disease through cellular self-digestion. Nature 451(7182):1069-1075 
Moreira P, Santos R, Zhu X, Lee H, Smith M, Casadesus G, Perry G (2010) Autophagy in Alzheimer's disease. Expert Rev Neurother 10:1209-1218

Nakatogawa H, Ichimura Y, Ohsumi Y (2007) Atg8, a ubiquitinlike protein required for autophagosome formation, mediates membrane tethering and hemifusion. Cell 130:165-178

Nixon RA (2007) Autophagy, amyloidogenesis and Alzheimer disease. J Cell Sci 120:4081-4091

Nixon RA, Wegiel J, Kumar A, Yu WH, Peterhoff C, Cataldo A, Cuervo AM (2005) Extensive involvement of autophagy in Alzheimer disease: an immuno-electron microscopy study. J Neuropathol Exp Neurol 64:113-122

Rami A (2009) Review: autophagy in neurodegeneration: firefighter and/or incendiarist? Neuropathol Appl Neurobiol 35:449-461

Ravikumar B, Duden R, Rubinsztein DC (2002) Aggregate-prone proteins with polyglutamine and polyalanine expansions are degraded by autophagy. Hum Mol Genet 11:1107-1117

Sarkar S, Rubinsztein DC (2008) Small molecule enhancers of autophagy for neurodegenerative diseases. Mol Biosyst 4:895-901
Soontornniyomkij V, Risbrough VB, Young JW, Wallace CK, Soontornniyomkij B, Jeste DV, Achim CL (2010) Short-term recognition memory impairment is associated with decreased expression of FK506 binding protein 51 in the aged mouse brain. Age Dordr 32:309-322

Stokin GB, Lillo C, Falzone TL et al (2005) Axonopathy and transport deficits early in the pathogenesis of Alzheimer's disease. Science 307(5713): 1282-1288

Valverde F (1998) Golgi atlas of the postnatal mouse brain. Springer/Wein, New York

Wang Y, Martinez-Vicente M, Krüger U et al (2009) Tau fragmentation, aggregation and clearance: the dual role of lysosomal processing. Hum Mol Genet 18:4153-4170

Wang Y, Martinez-Vicente M, Krüger U et al (2010) Synergy and antagonism of macroautophagy and chaperonemediated autophagy in a cell model of pathological tau aggregation. Autophagy 6:182-183

Young JW, Powell SB, Risbrough V, Marston HM, Geyer MA (2009) Using the MATRICS to guide development of a preclinical cognitive test battery for research in schizophrenia. Pharmacol Ther 122:150-202 